\title{
DEVELOPMENT OF AN RF CIRCUIT AMPLIFIER FED BY A LOW POWER NONLINEAR TRANSMISSION LINE
}

\author{
L. P. Silva Neto ${ }^{\xi}$, H. M. Moraes, J. O. Rossi' ${ }^{1}$, J. J. Barroso ${ }^{1}$, E. Rangel ${ }^{1}$, A. F. Conceição \\ Federal University of São Paulo, Av. Cesare Mansueto Giulio Lattes, 1201 \\ São José dos Campos, SP, Brazil
}

\begin{abstract}
Lumped nonlinear transmission lines have been studied for the generation of radio frequency signals in the range of the order of tens of $\mathrm{MHz}$ up to a few hundreds of $\mathrm{MHz}$ depending on the nonlinear element used in the LC line. The oscillations obtained at the output of these lines are applied in defense mobile platforms and communications systems. Low power nonlinear transmission lines use varactor diodes as nonlinear elements, which show a strong nonlinear effect with capacitance variation of the order of $90 \%$ at their P-N junction with the applied voltage, which is an excellent performance to obtain oscillations at the line output. However, these semiconductor devices operate at low voltage, producing small voltage modulation depth, low power, and consequently reduced signal range. Looking for increasing the voltage modulation depth of the signal generated with nonlinear transmission lines, this work developed a radiofrequency amplifier using a Metal Oxide Semiconductor Field Effect Transistor - MOSFET model RD06HVF1. A 30-section line using varactor diodes MV209 as nonlinear elements can work as an RF source to obtain oscillations with a frequency of 33.3 $\mathrm{MHz}$ at the line output. By means of SPICE simulations, it has been demonstrated that an amplifier circuit connected to the output of this varactor diode transmission line can produce an increase of the voltage modulation depth produced at line output from $10.7 \mathrm{~V}$ to $40 \mathrm{~V}$ approximately, thus allowing higher level power to electromagnetic wave propagation and consequently higher signal range. Experimental comparison using a PCB prototype with the corresponding simulation will be also shown.
\end{abstract}

\section{I.INTRODUCTION}

Radiofrequency waves have been used as a way of transmitting information a long time ago, for example broadcasting communication using amplitude modulation and frequency modulation. This system is composed by oscillator, modulator, filters, amplifiers and antennas [1]. In this work we use a low power nonlinear transmission lines NLTL as a source of radiofrequency signal. The
NLTL use linear inductors and nonlinear varactor diodes for soliton generation. Solitons have been investigated to be used in radar and military application. In Fig. 1 it is shown a NLTL composed 30 sections with each one using an inductor of $2.7 \mu \mathrm{H}$ and a varactor diode model MV209. The resistances $R_{L}$ and $R_{C}$ represent the loss in the terminals of inductors and capacitors respectively [2]. The radiofrequency oscillations obtained in the output of these lines have a low voltage modulation depth (VMD) of the order of $10.7 \mathrm{~V}$ using a load of $47 \Omega$.

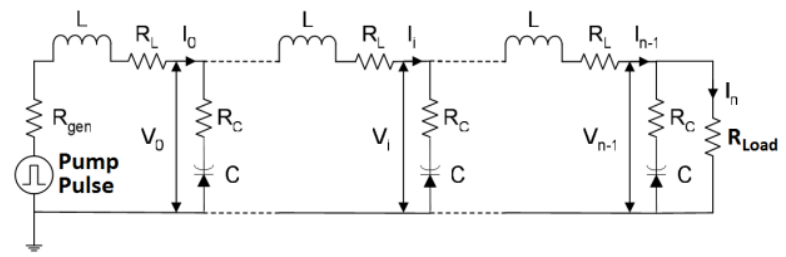

Figure 1. A standard varactor diode line used to produce $\mathrm{RF}$ in the $\mathrm{MHz}$ range.

In this work, we report the construction of a power amplifier using a MOSFET (Metal Oxide Semiconductor Field Effect Transistor) Technology to increase a Voltage Modulation Depth (VMD) of the soliton generated in the NLTL.

\section{METHODOLOGY}

MOSFET technology is widely used to make integrated circuit due to their faster switching and smaller size than bipolar junction transistor (BJT), allowing construction of devices (processors and memories) with reduced size. Other MOSFET technology characteristics are higher impedance input, of the order of some tens of mega ohms, better stability in temperature compared to BJT, and controlled by voltage, but its disadvantage is electrostatic voltage sensibility due silicon dioxide used to isolate the gate terminal to substrate [3]. In this work it was used a MOSFET model RD06HVF1 manufactured by Mitsubishi electric corporation [4]. This power MOSFET RD06HVF1 has been used in other works related to the RF amplification, for example, in an overhauser

\footnotetext{
$\xi$ email: lauro.paulo@unifesp.br

${ }^{1}$ National Institute for Space Research, Associated Plasma Laboratory, São José dos Campos, Brazil
} 
magnetometer RF power amplifier [5]. In Fig. 2 it is shown the polarization curve using $\mathrm{V}_{\mathrm{GS}}$ (Voltage across Gate to Source) higher than $5 \mathrm{~V}$. Note in the curve that below $5 \mathrm{~V}$ value there is no current from drain to source.

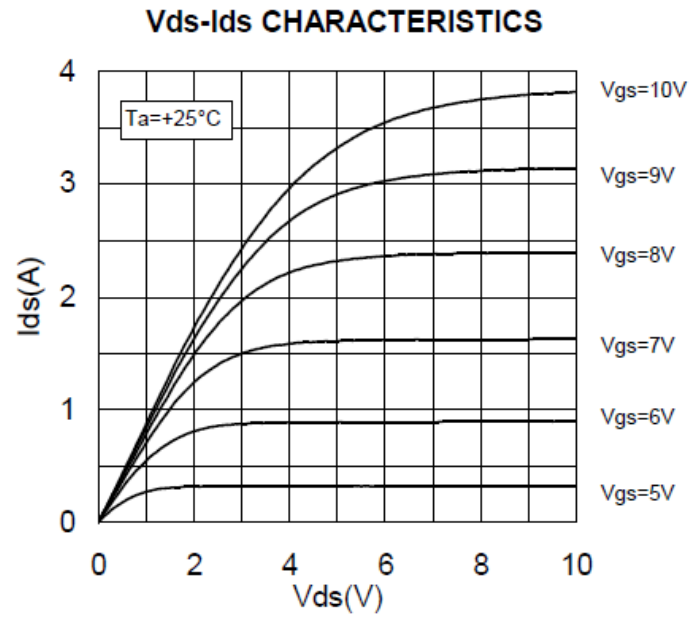

Figure 2. Polarization curve of the RD06HVF1 MOSFET [4].

Fig. 3 shows an experimental soliton wave generated by NLTL (see Fig. 1). In this graphic we can see that the oscillation peak is near to $16 \mathrm{~V}$, which means it can saturate the MOSFET when polarized with a DC signal. In this case, a pulse transformer was used to decrease the soliton wave at the MOSFET gate in such a way that the peak voltage of the soliton wave added to the DC polarization is below $20 \mathrm{~V}$ (avoiding the MOSFET saturation). In the Fig. 3 the soliton wave shows a voltage modulation depth (VMD) of the order of $10.7 \mathrm{~V}$ and generated frequency of $33.3 \mathrm{MHz}$ for a terminated $50 \Omega$ load.

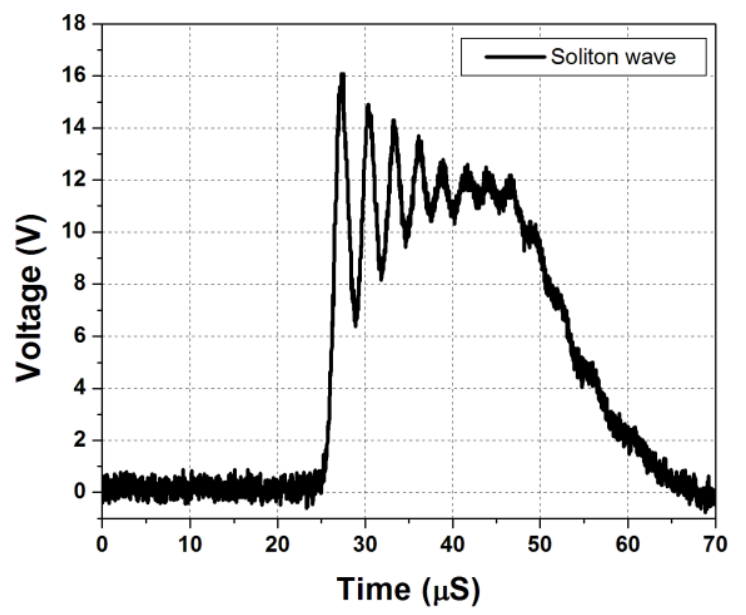

Figure 3. Soliton oscillations obtained at the NLTL output.

The electrical circuit used as soliton amplifier fed by an NLTL is shown in the Fig. 4.

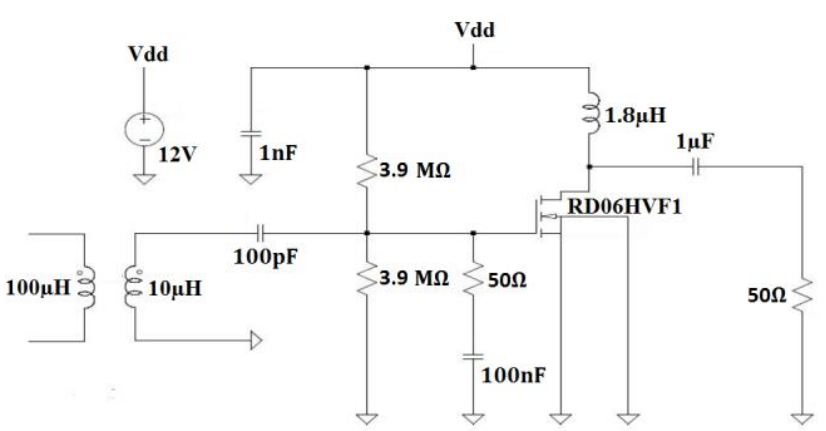

Figure 4. Power amplifier circuit using the MOSFET RD06HVF.

As discussed in the introduction section the NLTL uses a nonlinear component, in this case a varactor diode, which produces a nonlinear impedance $\mathrm{Z}(\mathrm{V})$ as a function of applied voltage, calculated by

$$
Z(V)=\sqrt{\frac{L}{C(V)}}
$$

where $\mathrm{L}$ is linear inductance and $\mathrm{C}(\mathrm{V})$ is the nonlinear capacitance as function applied voltage.

This nonlinear impedance is a challenge to polarize the MOSFET in the active region. The $\mathrm{Z}(\mathrm{V})$ variation produces an impedance mismatching to the power amplifier, meaning that the MOSFET will got to the cutoff or to the saturation regions. To solve this, we have used a pulse transform to isolate the NLTL from the power amplifier with a relation of order of 10:1 (represented by $100 \mu \mathrm{H}$ and $10 \mu \mathrm{H}$ in the electric circuit of Fig. 4) to ensure that the voltage does not increase above $20 \mathrm{~V}$ at the gate of amplifier.

The LC circuit tank formed by $1 \mathrm{nF}$ and $1.8 \mu \mathrm{H}$ are used to send a noise (high frequency) to ground, $3.9 \mathrm{M} \Omega$ resistors are used for the DC polarization of the MOSFET. Besides, $50 \Omega$ resistor in series with $100 \mathrm{nF}$ capacitor is used to improve stability of the circuit, $100 \mathrm{pF}$ and $1 \mu \mathrm{F}$ capacitors to decouple the $\mathrm{DC}$ signal of the radiofrequency (RF) wave. Finally, $50 \Omega$ represents the antenna load.

\section{RESULTS AND DISCUSSIONS}

Fig. 5 gives the simulated output soliton oscillations (red line) obtained with the NLTL coupled to the power amplifier, presenting a voltage modulation depth (VMD) of the order of $26.63 \mathrm{~V}$ and generated frequency of 33.3 $\mathrm{MHz}$ for a terminated $50 \Omega$ load. The LT-Spice software was used to simulate NLTL operation, by setting the pulse input amplitude at $10 \mathrm{~V}$, rise time near $10 \mathrm{~ns}$ and pulse duration of about $500 \mathrm{~ns}$ (see corresponding black line in Fig. 5). The NLTL output pulse is applied to the input of the amplifier as represented by the blue line in order to improve VMD on the output (see red line again). 


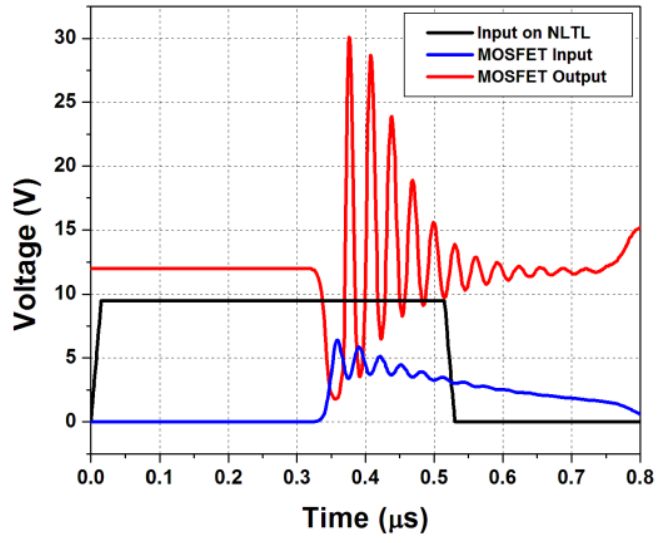

Figure 5. Simulation result obtained for the NLTL coupled to the RF power amplifier for a $50 \Omega$ load.

The experimental results using the same configurations and same parameters are shown in Fig. 6. We can see good approximation of input soliton for simulation and experiment, but experimental input has a distortion explained by destructive reflections due impedance matching. The input result is taken as an offset wave in the experimental output compared to the simulation, shown by the red line.

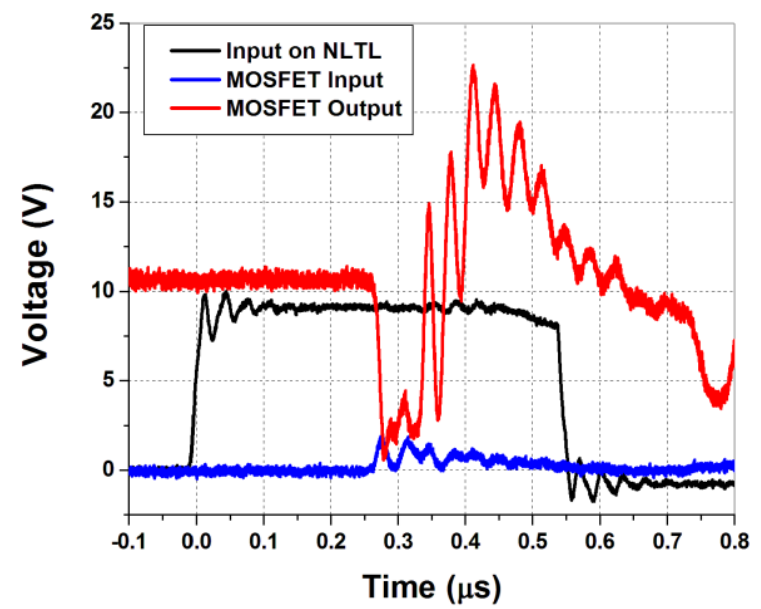

Figure 6. Experimental results obtained for the NLTL coupled to the RF power amplifier for a $50 \Omega$ load.

Table I summarizes the results showing the main parameters obtained such as number of oscillations and VMD produced at the amplifier input and output as well as the generated frequency and the gain of the amplifier in decibels. The amplifier gain was taken considering the VMD ratio between the input and output.

In the Tab. I, the VMD obtained at the output of the line before amplification was of the order of $10.7 \mathrm{~V}$. The simulation results at the amplifier output with a $50 \Omega$ load gives a VMD of about $26.63 \mathrm{~V}$, while the corresponding experimental one shows a VMD of the order of $12.07 \mathrm{~V}$. The results are of the order of $18.94 \mathrm{~dB}$ and $16.92 \mathrm{~dB}$ for simulation and experiment respectively.
Table I. Power amplifier parameters obtained from Figs.

5 and 6 using the MOSFET with a $50 \Omega$ load.

\begin{tabular}{|l|c|c|}
\hline \multicolumn{1}{|c|}{ Parameter } & Simulation & Experiment \\
\hline Input oscillation no. & 6 & 3 \\
\hline Output oscillation no. & 9 & 9 \\
\hline Frequency (MHz) & 33.3 & 31.2 \\
\hline Input VMD (V) & 3.01 & 1.72 \\
\hline Output VMD (V) & 26.63 & 12.07 \\
\hline Gain (dB) & 18.94 & 16.92 \\
\hline
\end{tabular}

The second configuration used a load of $1 \mathrm{k} \Omega$ and pulse width applied in the input of the NLTL of order $300 \mathrm{~ns}$ and the other parameters remained fixed. The simulation results are shown in Fig. 7.

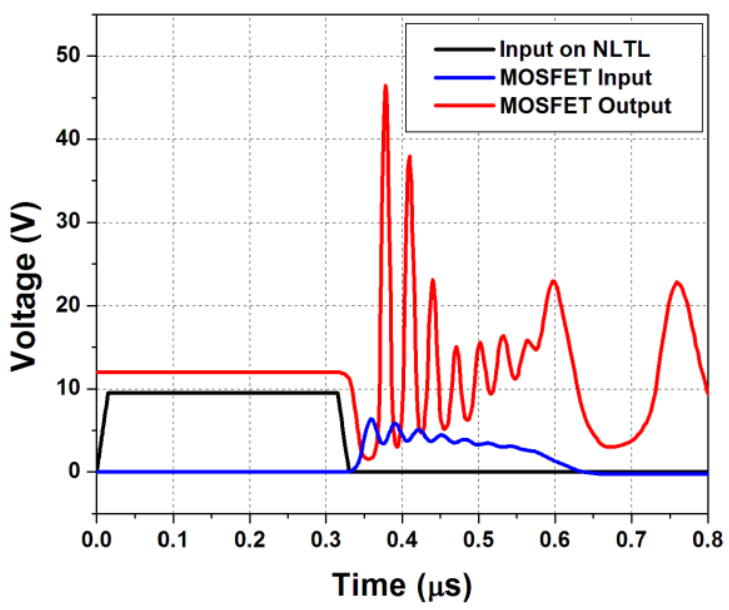

Figure 7. Simulation results obtained for the NLTL coupled to the RF power amplifier for a $1 \mathrm{k} \Omega$ load.

The experimental result using a load of $1 \mathrm{k} \Omega$ is the best one, showing a voltage modulation depth of the order of $43 \mathrm{~V}$ between second to third peaks as indicated by the red line in Fig. 8.

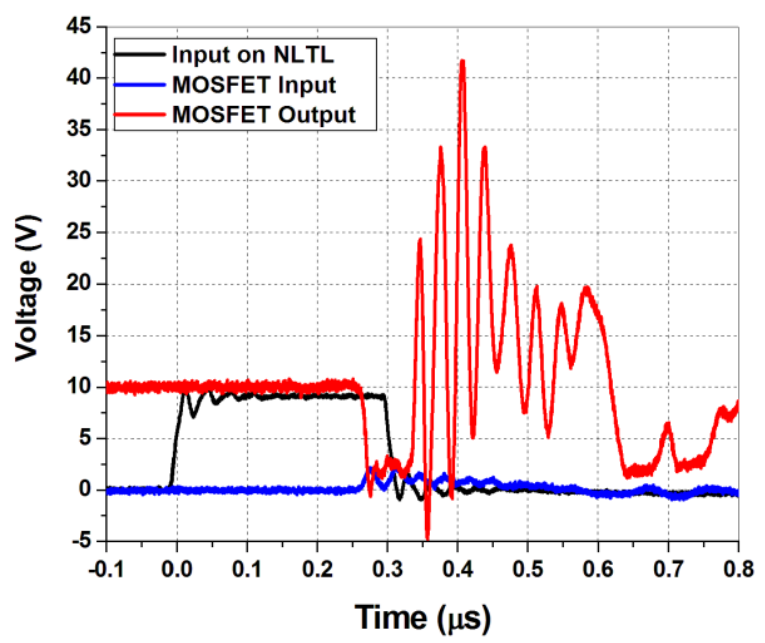

Figure 8. Experimental results obtained for the NLTL coupled to the RF power amplifier for a $1 \mathrm{k} \Omega$ load. 
Table II summarizes and compares simulation and experimental results for the main parameters investigated for the input/output waveforms obtained with a load of 1 $\mathrm{k} \Omega$.

In Tab. II, the simulation results after amplification using a $1 \mathrm{k} \Omega$ load gives a VMD of order of $43.53 \mathrm{~V}$, while the corresponding experimental one shows a VMD of the order of $36.81 \mathrm{~V}$. The simulated gain is of about $23.14 \mathrm{~dB}$ and the corresponding experimental gain measured is of the order of $25.56 \mathrm{~dB}$.

Table II. Power amplifier parameters obtained from Figs. 7 and 8 using the MOSFET with $1 \mathrm{k} \Omega$ load.

\begin{tabular}{|l|c|c|}
\hline \multicolumn{1}{|c|}{ Parameter } & Simulation & Experiment \\
\hline Input oscillation no. & 6 & 6 \\
\hline Output oscillation no. & 7 & 7 \\
\hline Frequency (MHz) & 33.3 & 33.3 \\
\hline Input VMD (V) & 3.03 & 1.94 \\
\hline Output VMD (V) & 43.53 & 36.81 \\
\hline Gain (dB) & 23.14 & 25.56 \\
\hline
\end{tabular}

\section{CONCLUSIONS}

In this work, it was shown that a MOSFET amplifier can increase the voltage modulation depth (VMD) of the soliton oscillations generated by a capacitive NLTL using varactor diodes as nonlinear elements. The main challenge of this wok was to match the NLTL varying impedance (increasing with the voltage) to the input impedance of the amplifier by means of a pulse transformer. Both experimental and simulation results presented soliton oscillations with better VMD, of higher gain and with an increased number of oscillations when using an RF amplifier with a higher terminated load. Better or higher VMD of the oscillations means that the soliton waves generated can propagate at longer distances if irradiated.

\section{REFERENCES}

[1] L. P. Silva Neto, J. O. Rossi, J. J. Barroso, E. Schamiloglu, "High- Power RF Generation from nonlinear transmission lines with barium titanate ceramic capacitors," IEEE Trans. Plasma Sci., vol. 44, no. 12, pp. 3424-3431, Dec. 2016.

[2] L. P. Silva Neto, J. O. Rossi, J. J. Barroso, E. G. L, Rangel and E. Schamiloglu, "Improving the Voltage Modulation Depth and RF power generated on Nonlinear Transmission Lines," in Proc. IEEE IPMHVC, 2018, pp. $416-420$.

[3] R. L. Boylestad and L. Nashelsky, "Electronic devices and circuit theory," $11^{\text {th }}$ ed., Pearson Education Inc., 2013.
[4] Mitsubishi Electric Corporation, "Silicon RF power MOSFET," RD06HVF1 Datasheet, Oct. 2011.

[5] D. Zheng, S. Zhang, X. Guo, H. Fu "An improved RF circuit for overhauser magnetometer excitation," in Proc. SPIE 9595, Radiation Detectors: Systems and Applications XVI, Aug. 2015, pp. 1-9. 